\title{
The AI Journey: The Road Traveled and the (Long) Road Ahead
}

\author{
Ramon Lopez de Mantaras \\ Artificial Intelligence Research Institute, \\ Spanish National Research Council (CSIC), Spain \\ mantaras@iia.csic.es
}

\begin{abstract}
In this talk I will first briefly summarize the many impressive results we have achieved along the road so far traveled in the field of AI including some concrete results obtained at the IIIA-CSIC. Next I will describe some of the future challenges to be faced along the (long) road we still have ahead of us with an emphasis on integrated systems, a necessary step towards humanlevel AI. Finally I will comment on the importance of interdisciplinary research to build such integrated systems (for instance, sophisticated robots having artificial cartilages, artificial muscles, artificial skin, etc) using some examples related to materials science.
\end{abstract}

